\title{
Designing website attributes to induce experiential encounters
}

\author{
Ming-Hui Huang* \\ Institute of Electronic Commerce, National Chung Hsing University, \\ 250 Kuo Kuang Road, Taichung 402, Taiwan
}

\begin{abstract}
Can websites be designed to be both utilitarian and hedonic? This article approaches this question by identifying Web attributes, their direct impacts on experiential flow, and their direct and indirect impacts on the utilitarian and hedonic aspects of Web performance. The results presented here support the proposal that, as an information-laden medium, a successful website must be able to use its attributes to satisfy both the information and entertainment needs of users.

(C) 2003 Elsevier Science Ltd. All rights reserved.

Keywords: Experiential flow; Utilitarian; Hedonic; Web performance; Web attributes
\end{abstract}

\section{Introduction}

The Web is evolving into an environment that caters for a range of activities, including entertainment, exploration, communication, and learning. Users not only search the Web for specific information, they also surf it for entertainment, stimulation, and to socialize. The search for specific information is the result of goaldirected need, whereas surfing the Web for other purposes is the result of the experiential need of users (Eliashberg \& Sawhney, 1994; Hoffman \& Novak, 1996; Novak, Hoffman, \& Yung, 2000; Wolfinbarger \& Gilly, 2001). Users use and look for different Web attributes depending on their needs (Singh \& Dalal, 1999). The ability to create and manage websites that fulfill one or more of the needs of users will become a distinguishing feature of successful e-business.

The utilitarian function of websites in meeting goal-directed need is well documented. This article considers the other side of the coin: can websites be designed to

* Tel./fax: $+886-4-22854910$.

E-mail address: mhhuang@nchu.edu.tw (M.-H. Huang). 
generate an experiential experience and to enhance hedonic performance? To address this issue, we examined numerous Web attributes and divided these attributes into relevant components such as complexity, novelty, and interactivity. We also investigated the individual and collective impacts of these properties on experiential flow, an intrinsically enjoyable experience that some believe is an essential feature of commercially compelling websites (Novak et al., 2000). The Web attributes and flow experience were further linked to both the utilitarian and hedonic aspects of Web performance. Because the Web mixes goal-directed and experiential behavior, our results can be used to develop and evaluate websites in terms of the extent to which they satisfy these two needs.

\section{The concepts}

\subsection{Web attributes: complexity, novelty, and interactivity}

Attributes are features or aspects of a website. Users see each website as a bundle of attributes with varying capacities to satisfy their needs. Attributes can be technology or user-oriented. Technology-oriented attributes are the structural properties of a site such as hyperlink multimedia modalities, whereas user-oriented attributes are the qualitative experiences of users in relation to the structural properties of a site, for example navigability and demonstrability.

We investigate the user-oriented attributes of websites, because they have the greatest influence on user decision-making. Although we do not explicitly consider the structural properties of websites, the user-oriented approach includes within it user preferences in regard to structural properties such as multidimensionality, participation, richness, and demonstrability. Drawing upon information theory, the concept of information rate is used to gauge the complex spatial and temporal arrangement of stimuli in a website setting (Mehrabian \& Russell, 1974). The spatial and temporal arrangement of attributes varies from site to site. These attributes can be described as multidimensional, rich, selective, multiple, large-scale, broad, active, responsive, interactive, participatory, dynamic, demonstrable, imaginary, surprising, innovative, new, flexible, or multimedia.

Complexity and novelty have been identified as the two major categories for attributes in physical settings (Berlyne, Craw, \& Salapatek, 1963; Donovan \& Rossiter, 1982; Hwang \& Lin, 1999; Iselin, 1988). Nevertheless, the attributes of a website cover a larger variety of categories than those of traditional media. Interactivity has emerged as a critical attribute in computer-mediated communications, because it is seen as the key advantage of the medium (Ha \& James, 1998). Thus, we identified complexity, novelty, and interactivity as the three broad categories for website attributes:

\subsubsection{Complexity}

Complexity refers to the amount of information that a site is perceived to offer, including aspects such as the number of alternatives, the number of attributes, and the variation in the information provided by the attributes (Berlyne et al., 1963; 
Campbell, 1988; Donovan \& Rossiter, 1982; Hwang \& Lin, 1999; Iselin, 1988). Both the topical range and structural properties of a website contribute to how it is perceived. For example, sites that convey information through a broad range of text and graphics in a multidimensional format are likely to be perceived as complex.

\subsubsection{Novelty}

Novelty can take the form of novel experiences or information, or both in combination (Bianchi, 1998). It refers to the aspects of website attributes that users find unexpected, surprising, new, and unfamiliar. Novelty can be created by freshness of content and innovation in information technology. Novelty is often conceptualized as the opposite of familiarity, and is associated with a lack of experience with the website or its offerings (Berlyne et al., 1963). Many Web technologies provide attributes that are novel to users, such as multimedia modalities and the simulation of humanlike characteristics.

\subsubsection{Interactivity}

Interactivity describes the extent of information exchange between a website and its users. Interactivity is the attribute that most distinguishes websites from other media. Although the meaning of interactivity may seem self-evident, the term has been applied to widely divergent phenomena and has been defined in a number of ways. Synthesizing the results from the literature (see Fig. 1), the following core conceptual domains were identified for interactivity (Burgoon, Bonito, Bengtsson, \& Ramirez, 1999/2000; Ha \& James, 1998). Also given are examples of technology attributes corresponding to these conceptual domains.

- Responsiveness: the degree to which a site is perceived to respond to users' needs. An e-mail management system is one such technology attribute showing responsiveness.

- Individualization: the extent to which a site is perceived to provide information personalized to the unique needs of each user. For example, the use of information-filtering agents to provide users with personalized information achieves this purpose. We see this in popular sites such as My Yahoo! and My Dell.

- Navigability: the extent to which a site is perceived to have unrestrained connectedness, including links to other parts of the site and to other sites to allow easy information retrieval. For site visitors, hypertext in websites can create a feeling of being connected to the world by allowing visitors to jump, with minimal effort, from one point in cyberspace to another.

- Reciprocity: the extent to which a site is perceived to provide two-way information exchange between the site and users. A reciprocal loop can be formed by a company providing information to users at a website along with a way for users to respond to this information. Posting answers to FAQs is one example of reciprocity.

- Synchronicity: the extent to which users feel that they have real-time bidirectional feedback. With a higher degree of synchronicity, the information 


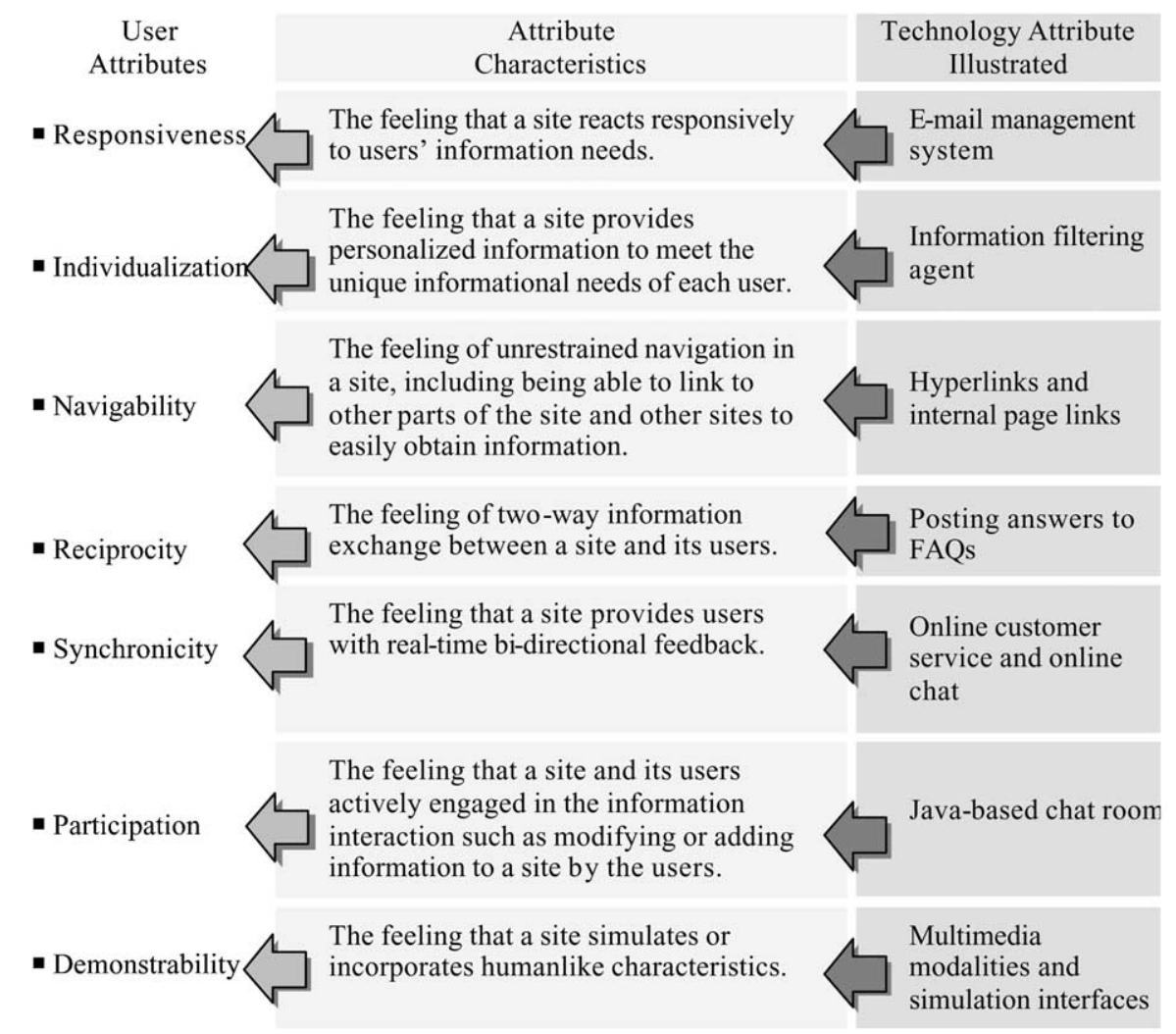

Fig. 1. User and technology attributes of interactivity illustrated.

provided is more immediately available to users. Online customer services and online chat make synchronicity possible.

- Participation: the extent to which a site and its users actively interact. For example, a site may allow visitors to modify or add information to the site. When users are given the opportunity to create content, information exchange is facilitated.

- Demonstrability: the extent to which a site is perceived to simulate or incorporate humanlike characteristics. Demonstrability reflects the media richness of a site. It is influenced by whether a site format utilizes one or more modalities such as text, audio, visual, or touch, and the extent to which a site supports a variety of symbols to present diverse information.

\subsection{Experiential flow}

According to the theory of flow, experiential flow is the optimal and enjoyable experience in which we feel "in control of our actions, masters of our own fate...we 
feel a sense of exhilaration, a deep sense of enjoyment" (Csikszentmihalyi, 1990, p. 3). Applying the concept of optimal flow to the Web environment, flow is a subjective human and computer-mediated interaction experience, representing the user's perception of the interaction with a site as playful and exploratory (Hoffman \& Novak, 1996; Novak et al., 2000). The consideration of flow is important for understanding user behavior on the Web. The virtual hypermedia environment of the Web incorporates various types of interactivity (human-human, human-computer, and computer-computer) and possesses unique characteristics which distinguish it from the physical world (Hoffman \& Novak, 1996). This interactivity and its distinction from everyday activities may provide Web users with an environment in which to experience flow (Chen, Wigand, \& Nilan, 1999).

The overall flow experience can be divided into four components: (1) the perception of a sense of control over the computer interaction, (2) the degree to which the user's attention is focused on the interaction, (3) the curiosity aroused during the interaction, and (4) the extent to which the user finds the interaction intrinsically interesting (Trevino \& Webster, 1992; Webster, Trevino, \& Ryan, 1993). The four components reveal that flow is both cognitive and affective. It represents the optimal experience that stems from people's perceptions of challenges and skills in given situations (Csikszentmihalyi, 1975); the experience of flow requires a Web user to meet challenges imposed by Web environments (Hoffman \& Novak, 1996). In the flow state, the computer user concentrates on the activity to such an extent that they have little attention left to consider anything else (Ghani \& Deshpande, 1994; Hoffman \& Novak, 1996; Novak et al., 2000). Flow is fun (Privette, 1983). The state of mind that results from achieving flow is extremely gratifying (Novak et al., 2000) and self-motivating (Trevino $\&$ Webster, 1992). When in a flow state, an individual finds the activity intrinsically interesting (Csikszentmihalyi, 1975). Flow is most often experienced in activities that reward the participants (Csikszentmihalyi, 1975), for example the immediate feedback provided by computers imparts enjoyment to users (Webster et al., 1993).

\subsection{Utilitarian and hedonic aspects of Web performance}

Users visit websites not only for information, but also for entertainment. Through the inclusion of attributes that cause users to perceive a site as abundant, interactive, or novel, a website can be created that provides users with opportunities to experience flow and that meets both their utilitarian and hedonic needs.

We identified two aspects of Web performance: utilitarian and hedonic. The utilitarian aspect of Web performance is the evaluation of a website based on the assessment by users regarding the instrumental benefits they derive from its nonsensory attributes. It is related to the performance perception of usefulness, value, and wisdom (Batra \& Ahtola, 1990). Utilitarian performance results from user visiting a site out of necessity rather than for recreation; therefore, this aspect of performance is judged according to whether the particular purpose is accomplished (Davis, Bagozzi, \& Warshaw, 1992; Venkatesh, 2000).

The hedonic aspect of Web performance is the evaluation of a website based on the assessment by users regarding the amount of fun, playfulness, and pleasure they 
experience or anticipate from the site. It reflects a website's entertainment value derived from its sensory attributes, from which users obtain consummatory affective gratification (Batra \& Ahtola, 1990, Crowley, Spangenberg, \& Hughes, 1992). A website performs well in the hedonic aspect when users perceive the site to be enjoyable in its own right, apart from any performance consequences that may be anticipated (Davis et al., 1992; Igbaria, Schiffman, \& Wieckowski, 1994; Venkatesh, 2000).

\section{Methodology}

\subsection{Procedure}

The data for the study were collected in May and June 2000 through a questionnaire survey of Web users with varied demographic profiles and Web usage levels. The initial questionnaire was generated from existing academic and practitioner-oriented literature in electronic commerce and marketing. It was tested on Web users who either worked for companies in information management related positions or who studied in management related fields. The research team contacted more than 400 Web users through their academic and continuing education programs and obtained 243 valid questionnaires.

Each Web user was first asked to rate Web descriptors according to how appropriately they described the characteristics of the websites that they most frequently visited. This approach covers all types of websites, an important requirement for any attempt to construct a general description of Web attributes. The fact that these websites are the most frequently visited by the user suggests that they successfully meet the user's needs. After rating the descriptors, each user was then asked to indicate his or her flow state in the site and evaluate its performance.

\subsection{Measures}

The final questionnaire consisted of 37 items for $15 \mathrm{Web}$ attributes, 12 experiential flow measures, and $10 \mathrm{Web}$ performance measures (see Appendix). First, the Web characteristics were described by 15 descriptors capturing the complexity, novelty, and interactivity attributes. The 15 descriptors were purified from $58 \mathrm{Web}$ attributes on the basis of the results of exploratory and confirmatory factor analyses. The complexity attribute contained the items multidimensionality, richness, multiplicity, large size of scale, and breadth. The novelty attribute included the items imagination, surprise, innovation, and novelty. Finally, the interactivity attribute was composed of items such as activity, responsiveness, interactivity, participation, dynamics, and demonstrability. These attributes represent the attributes of websites as perceived by their users, and are potentially useful cues for users and website designers.

Second, a 12-item Likert flow scale was used to measure the experiential flow in the site (e.g. "This website allowed me to control the computer interaction" or 
"When navigating through this website, I was aware of distractions") (Webster et al., 1993). This scale contains four components with three questions per component. Responses were scored on seven-point scales ranging from strongly disagree (1) to strongly agree (7).

Finally, the evaluation of the site performance was measured using a 10-item twofactor 7-point semantic differential Web performance scale. The 10 items were selected from a pool of 42 candidates, which was collected through a review of the relevant literature, and on the basis of the results of confirmatory factor analyses. This scale is useful for assessing the competitive advantages of websites and the benefits derived from them. Evaluative criteria such as order, wisdom, reliability, effectiveness, and correctness were useful for determining the utilitarian aspects of Web performance, whereas evaluative criteria such as pleasant, nice, entertaining, agreeable, and soothing provided an assessment of the hedonic Web performance.

\subsection{Analysis}

We used structural equation modeling, which is more powerful than a simple correlation, to analyze the link between Web attributes, experiential flow, and Web performance (see Fig. 2). In the model, Web attributes were assumed to have direct impacts on experiential flow, and have direct and indirect impacts on the utilitarian and hedonic aspects of Web performance. Specifically, users are hypothesized to judge Web performance based on the Web attributes and on whether they experience flow in the site.

In addition to examining the overall measure of flow, an analysis was also conducted using the four components of flow (see Fig. 3). In this model, the direct impacts of Web attributes on Web performance were omitted from the analysis for simplicity. Significant impacts $(P<0.1)$ are reported.

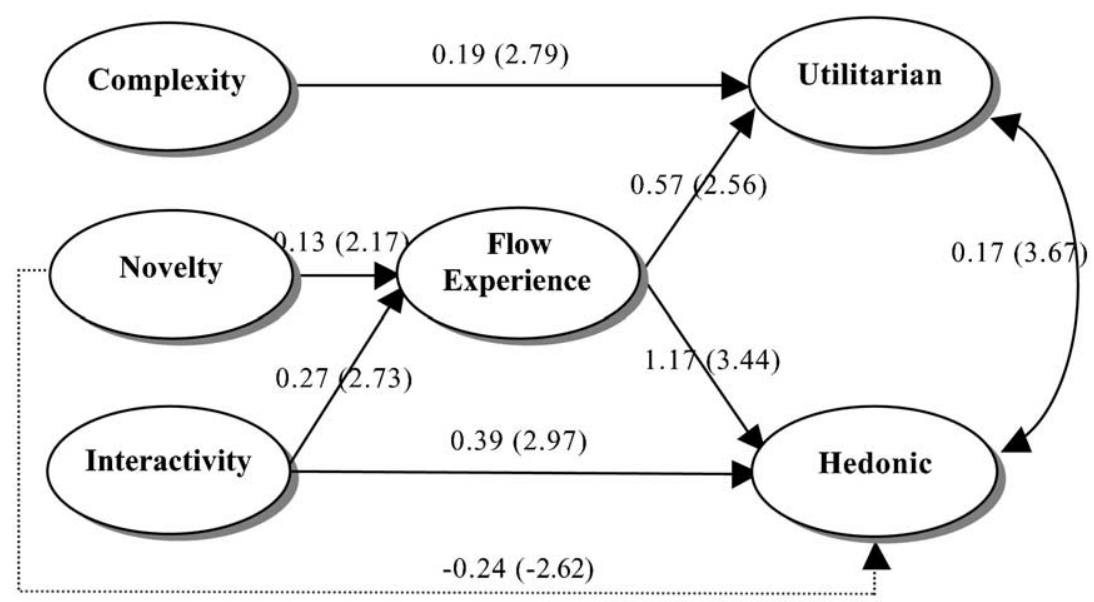

Fig. 2. The direct and indirect impacts of Web attributes on the utilitarian and hedonic aspects of Web performance, treating flow as an overall experience. $\rightarrow$ Positive impact; $\rightarrow \rightarrow$ Negative impact; $\leftrightarrow$ Correlation. Path coefficients and $t$-values are reported. 


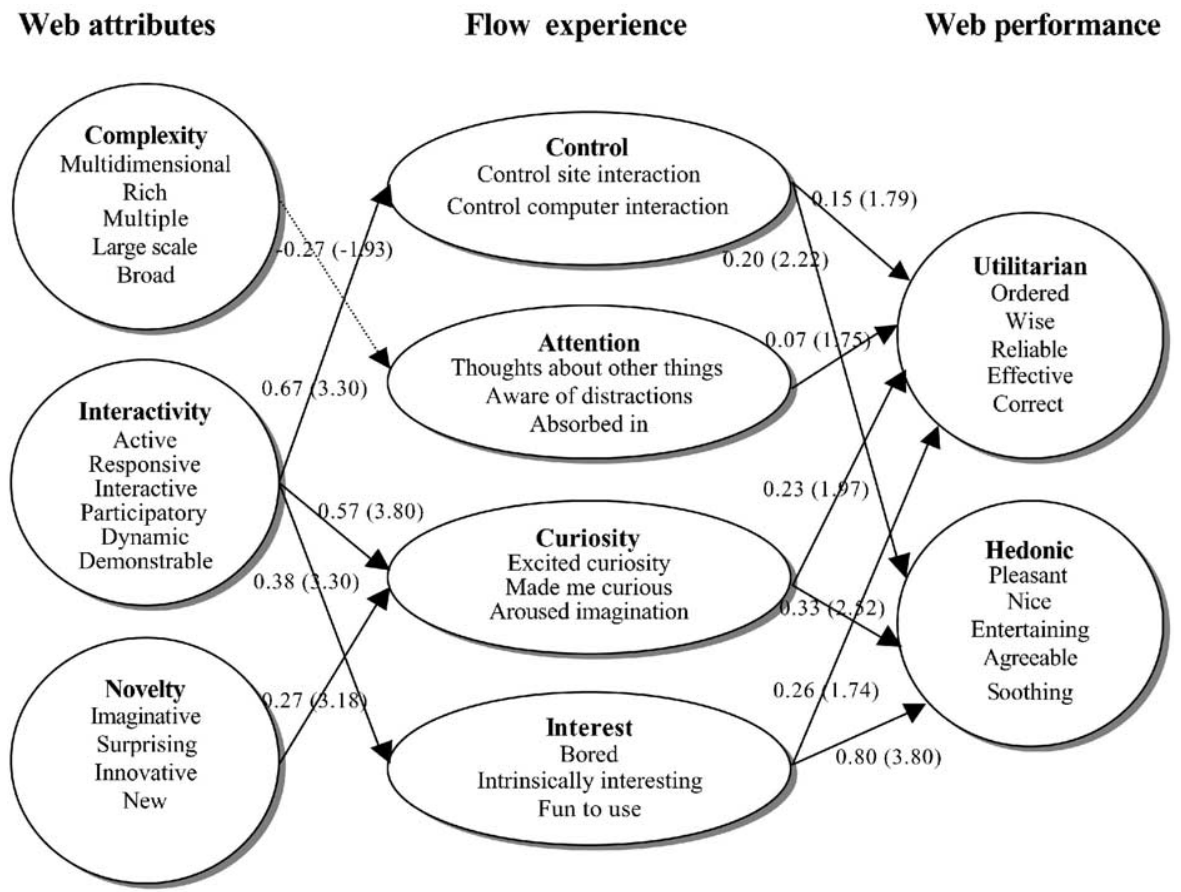

Fig. 3. The direct impacts of Web attributes on the experiential flow, and the indirect impacts on the utilitarian and hedonic aspects of Web performance. $\rightarrow$ Positive impact;...$\rightarrow$ Negative impact; $\leftrightarrow$ Correlation. Thick arrows denote the paths being significant at $0.05(\mid t$-value $\mid>1.96)$, while thin arrows denote the paths being significant at $0.1(\mid t$-value $\mid>1$.64). Path coefficients and $t$-values are reported.

\section{Results}

\subsection{Descriptive statistics}

The majority of the Web users were male (59.3\%) with ages ranging from 18 to 51 . The user profile was in agreement with the regular Georgia Tech Graphics, Visualization \& Usability (GVU) survey distribution of males and females (Bellman, Lohse, \& Johnson, 1999). The users surveyed tended to spend more than $3 \mathrm{~h}$ per week on the Web (74.6\%), with $24 \%$ spending more than $10 \mathrm{~h}$ per week. Users most commonly use the Web for a combination of work and entertainment $(75.3 \%)$. The websites that they most frequently visited fell primarily into the categories Entertainment (19.2\%), News and Media (18.5\%), Business and Economy (16.6\%), Reference (11.3\%), and Computers and Internet (9.9\%).

\subsection{Major findings}

The results of the survey are fourfold. First, we used accepted psychometric techniques to identify viable Web attributes and aspects of Web performance. From the 
confirmatory factor results we captured the existence of three broad categories of Web attributes, designated as complexity, novelty, and interactivity. In total these three broad sets consisted of 15 specific attributes associated with the most frequently visited websites (see Appendix). The reliability and variance extracted (in brackets) for complexity, novelty and interactivity were $0.80(42.9 \%), 0.67(34.1 \%)$, and $0.70(28.4 \%)$ respectively. The dimensionality of the site performance scale was similarly confirmed. The reliability and variance extracted (in brackets) for utilitarian and hedonic performance were $0.86(54.6 \%)$ and $0.89(62.6 \%)$ respectively.

Second, results of confirmatory factor analyses for the flow scale suggested the omission of one unreliable statement, leaving 11 questions representing Control, Attention, Curiosity, and Interest. The reliability and variance extracted (in brackets) for control, attention, curiosity, and interest were $0.68(52.5 \%), 0.82(60.6 \%)$, $0.71(45.2 \%)$, and $0.72(48.3 \%)$, respectively.

Third, treating flow as an overall experience results of the structural equation modeling analysis showed that Web attributes are linked to experiential flow differentially, and can meet both goal-oriented and experiential needs directly (see Fig. 2). Complex websites are deemed by users to be useful (coefficient $=0.19, t=2.79$ ). Interactivity is the key to creating experiential flow (coefficient $=0.27, t=2.73$ ) and is the most important attribute for determining hedonic Web performance (coefficient $=0.39, t=2.97$ ). Novelty is contingent in that it facilitates flow (coefficient $=0.13, \quad t=2.17$ ), but undermines hedonic Web performance (coefficient $=-0.24, t=-2.62$ ). Analysis of the components of flow (see Fig. 3) showed that complexity distracted attention (coefficient $=-0.27, t=-1.93$ ), novelty excited curiosity (coefficient $=0.27, t=3.18$ ), and interactivity increased control (coefficient $=0.67, t=3.30$ ), curiosity (coefficient $=0.57, t=3.80$ ), and interest (coefficient $=0.38, t=3.30$ ).

Fourth, experiential flow was found to enhance both utilitarian (coefficient $=0.57$, $t=2.56$ ) and hedonic (coefficient $=1.17, t=3.44$ ) Web performance (see Fig. 2). Thus, our study establishes the following relationship between online user experience and online marketing outcome variables: the more profoundly users are in a flow state during their site visits, the more likely they are to consider the site as useful and pleasant. Consideration of the components of flow (see Fig. 3) showed that attention is utilitarian (coefficient $=0.07, t=1.75$ ), control (coefficient $=0.20$, $t=2.22$ ) and interest (coefficient $=0.80, t=3.80$ ) are more hedonic than utilitarian (shown in thick arrows pointing to the hedonic performance), and curiosity is both utilitarian (coefficient $=0.23, t=1.97$ ) and hedonic (coefficient $=0.33, t=2.52$ ). The different components of experiential flow can be used strategically for specific management purposes.

\section{Discussion}

What are the implications for management and research of these findings? By identifying the major Web attributes, assessing their connection to experiential flow, and linking both to utilitarian and hedonic Web performance, this article presents 
the key features influencing information seekers and entertainment surfers and thus facilitates the process of designing and evaluating websites.

\subsection{Using Web attributes to induce experiential flow}

The present results show that complexity distracts attention, novelty excites curiosity, and interactivity increases control, curiosity, and interest. These findings suggest the following strategic use of Web attributes to induce experiential flow.

\subsubsection{Complexity distracts attention}

We found that complexity tends to distract users from relevant information. When faced with an abundance of information, users feel unable to absorb it, are more easily distracted, and think about other things during site navigation. Users may be overwhelmed by the perceived complexity of a website, and consequently find it difficult to concentrate. Our results are consistent with the information overload perspective, which suggests a negative relation between complexity and flow (Hoffman \& Novak, 1996; Hwang \& Lin, 1999). Users often feel stressed when required to deal with excessive information. When users are confronted by a site offering a wide variety of options, they may be overwhelmed and believe that their skills are inadequate for meeting the challenges posed by the site. Complexity represents a challenge to users' cognitive capacity; thus, users often perceive a website with too much information to be unpleasant. For this reason, "keeping it simple" is an acknowledged value-adding practice that is used by leading e-businesses such as FedEx, Alamo, Direct Line and Dell Excel to make it easy for customers to do business with them (Willcocks \& Plant, 2001).

\subsubsection{Novelty excites curiosity}

We found that novelty can act as a curiosity generating mechanism that arouses the imaginations of users and captures their interest in a site. Similar findings were obtained elsewhere. In a study of catalog shopping, Stell and Paden (1999) found that novel stimuli arouse the curiosity of readers and motivate them to examine the catalog information further. Novelty is thought to be an innate human preference, and varied, novel, and surprising stimuli can elicit sensory curiosity (Bianchi, 1998). Users gain excitement and pleasure from seeking out new things. Therefore, incorporating novel elements into a website can attract curious users and bring out the enjoyable experience of flow.

\subsubsection{Interactivity increases control, curiosity, and interest}

We found that interactivity has a consistent positive impact on the control, curiosity, and interest components of flow, but has little effect on the attention component. Similar findings have been reported by Novak et al. (2000), who found that higher levels of interactivity are not associated with more focused attention. In summary, interactivity enhances the subjective feeling of "having control" over the interaction with the site, excites the curiosity of users, and makes navigation intrinsically interesting. 
Interactivity is considered an important determinant of experiential flow (Novak et al., 2000). Interactive Web attributes increase "the degree to which participants in a communication process can exchange roles and have control over their mutual discourse" (Rogers, 1995). Because the Web is a computer-driven environment, it can perform a variety of interactive input-output functions. Creating sites that can receive and respond to input from users is the essence of Web interactivity, because the interactivity offered by such sites not only enhances users' sense of control in a manner that is not possible with more static technologies (Ha \& James, 1998), but also enhances and alters the entertainment experience of users (Bryant \& Love, 1996). Functionalities such as e-mails, questions and answers on a website can provide users with such an interactive environment. For example, the interactive Web activities of users of Internet discussion groups, including information retrieval on the Web, reading and posting on newsgroups, and reading and replying to e-mail, have been found to be associated with flow during Web use (Chen et al., 1999).

\subsection{The consequences of flow: utilitarian or hedonic?}

The results of our study indicate that flow elicits favorable Web evaluations for both the utilitarian and hedonic aspects. Flow was found to have a stronger impact on the hedonic aspect, as reflected in the greater number of components of flow that are linked to hedonic performance (see the thick arrows in Fig. 3).

The components of flow naturally distinguish themselves into the four categories cited above, with attention facilitating utilitarian performance, curiosity facilitating both the utilitarian and hedonic aspects of Web performance, and control and interest exerting greater influence over hedonic performance. This suggests that flow can be both utilitarian and hedonic, depending on the sources of the flow experience.

\subsubsection{Attention is utilitarian}

We found that attention is a significant facilitator of utilitarian Web performance. Attention is a series of activities in which users selectively allocate cognitive resources (Kahneman, 1973). Because human cognitive resources are limited, it is impossible for users to process all of the information available at any given moment. Consequently, the cognitive system is constantly selecting information for further processing. Users use the attention mechanism to control the choice of stimuli that will be allowed, in turn, to control his or her behavior. Retaining the attention of users is therefore essential. Users who are completely absorbed in navigating the Web are more likely to retain what they perceive than users who are not. Attention facilitates the learning needed to progress to instrumental usage involving browsing and searching (Hoffman \& Novak, 1996; Novak et al., 2000; Webster et al., 1993).

\subsubsection{Curiosity is both utilitarian and hedonic}

The curiosity component of flow is a facilitator of both utilitarian and hedonic Web performance. Curiosity is seen as a motivational variable that represents desire for information (Loewenstein, 1994). It can be an extrinsically motivated desire for information to resolve uncertainty (Loewenstein, 1994). This type of information 
seeking is the goal-directed Web use that treats the acquisition of information as a means to some further end (Steenkamp \& Baumgartner, 1992). Curiosity can also be an intrinsically motivated desire for information that is not driven by a utilitarian goal (Posnock, 1991). This type of information seeking is the experiential Web use that treats the acquisition of information as an end in itself (Steenkamp \& Baumgartner, 1992). The two sources of curiosity and their differential linkages to the two aspects of Web performance suggest that the outcome (the utilitarian aspect) and the process (the hedonic aspect) of information acquisition are both relevant to Web performance. Web users may examine and explore websites in which they have little interest simply because their curiosity is aroused by the characteristics of the sites.

\subsubsection{Control and interest are more hedonic}

The control and interest components are dual-facilitators of Web performance, although they have a greater impact on hedonic performance than on utilitarian performance.

Control implies the freedom to act on the Web. Users who feel that they have control over the human and computer interaction, and who feel in control of actions and final choices, feel more confident than users who do not feel in control. In a study of Internet discussion groups, Web users reported that they felt a sense of control when engaged in Web activities such as navigation and searching (Chen et al., 1999). These Web activities not only enhance the sense of control, but also increase the enjoyment of Web users.

In experiential Web use, users are intrinsically interested in the process of navigation, not the outcome of navigation. Users seek flow primarily because it brings them enjoyment. For example, Hoffman and Novak (1996) suggested that Web surfers exploring the Web in their daily quest for the latest interesting sites do so in an intrinsically motivated experiential flow state. It represents the intrinsic motivation of users toward "playfulness" and "having fun" during computer interactions, which is related to feelings of pleasure and satisfaction derived from websites (Venkatesh, 2000).

\section{Implications}

\subsection{Three keys to inducing experiential encounters}

This article lends credence to the use of Web attributes to induce experiential encounters. The empirical results verify that websites can be designed with attributes that shape experiential flow, and both the Web attributes and this experiential flow determine the utilitarian and hedonic Web performance.

\subsubsection{Interactivity is the key}

Interactivity is a critical concept in computer-mediated communications, because it is the key advantage of the medium (Burgoon et al., 1999/2000; Ha \& James, 1998). Despite its importance in Web management, the effective use of interactivity 
to enhance user evaluations of Web performance and, more importantly, to adjust specific aspects of performance, remains a challenge. The importance of interactivity in inducing experiential counters suggests that companies can reap greater benefits from Web technology by making better use of interaction attributes in website design. To increase interactivity, websites should be designed to be active, responsive, interactive, participatory, dynamic, and demonstrable. Websites that form part of the "online community" are highly interactive in terms of these interactivity attributes, and the emulation of the interactivity offered by such sites is advocated for successful e-business (Williams \& Cothrel, 2000).

To build on the present results, empirical studies should be carried out to examine the links between the technology-oriented attributes of interactivity (e.g. Java-based chat room) and the user-oriented attributes of interactivity (e.g. participation). There are numerous technology attributes in addition to those illustrated in Fig. 1 that users may perceive as interactive. Users may prefer some technology attributes over others. Hence, empirical testing of the degrees of interactivity of technology attributes based on user perceptions should be carried out to guide the design of websites that successfully induce experiential encounters on the Web.

\subsubsection{The significance of satisfying hedonic need}

Managers assessing the performance of websites should attempt to measure both the utilitarian and hedonic aspects that in conjunction with Web attributes and experiential flow, can be useful in making managerial decisions. Web managers can design websites to achieve either utilitarian or hedonic performance, or both. Further, the stronger impact of flow on hedonic Web performance suggests that more attention should be paid to online experiential behavior as an additional communication path to customers. The positive correlation between the utilitarian and hedonic aspects of Web performance means that a site that satisfies one aspect will bring out the other aspect, increasing the overall satisfaction.

The precise nature of the positive relationship between the two aspects of Web performance should be pursued in future studies. Two possibilities have been suggested for the association between the utilitarian and hedonic aspects of Web performance. One possibility is that the two aspects are parallel and correlated, but not causally related. For example, Hammond, McWilliam, and Diaz (1998) found that Web users with a hedonic task did not value the fun aspects of the Web more than those with a utilitarian task, and that Web users with a utilitarian task did not value the information aspects of the Web more than those with a hedonic task. This view implies that the two aspects are equally and simultaneously important in meeting Web users' needs. The other possibility is that the utilitarian and hedonic aspects are two consequential, causally related stages of Web performance, with the occurrence of one leading to the other. Srinivasan (1987) viewed the two aspects as forming two distinct but consecutive stages of the consumer choice process, where product, personality, and context determine the aspect that dominates this process. Albers-Miller and Stafford (1999) argued that compared with utilitarian services, experiential services are more conspicuous, and more likely to be perceived as an extension of the consumer. This view suggests that the hedonic performance is an extension of the 
utilitarian performance; therefore, utilitarian performance can bring about hedonic performance, contributing to total satisfaction.

\subsubsection{Web attributes and flow interplay}

Web attributes can be used not only to aid users in making decisions, but also as tools to enhance online experiential enjoyment. Providing a large amount of information without considering aspects such as interactivity and novelty can lead to distracting and disadvantageous results. Novelty can serve as a mechanism for exciting curiosity to create initial success, and interactivity can then generate the full experience of flow. Using the competitive advantage of the Web, experiential flow can be created, and both utilitarian and hedonic needs can be satisfied. These results provide managerial tools for Web strategies and suggest that the proper use of Web attributes can deliver the greatest satisfaction to users.

Future research should delve into the relationships between Web attributes. Such research should investigate questions such as how interactivity can be used to reduce the perceived complexity of websites, and how novelty can be handled through the manipulation of the other two attributes.

\subsection{Concluding remarks}

Can websites be designed to be both utilitarian and hedonic? This article approaches this question by identifying Web attributes, their direct impacts on experiential flow, and their direct and indirect impacts on the utilitarian and hedonic aspects of Web performance. The results presented here support the proposal that, as an information-laden medium, a successful website must be able to use its attributes to satisfy both the information and entertainment needs of users.

\section{Appendix. The abbreviated questionnaire}

\section{The Web attributes measure}

Please rate the following Web descriptors for appropriateness in describing Web characteristics on the basis of the website you most frequently visited.

\section{Complexity}

1. Multidimensional

2. Rich

3. Multiple

4. Large scale

5. Broad 
Interactivity

1. Active

2. Responsive

3. Interactive

4. Participatory

5. Dynamic

6. Demonstrable

Novelty

1. Imaginative

2. Surprising

3. Innovative

4. New

The flow measure

Please describe your navigation experience in your most frequently visited website by indicating your level of agreement using the following scale.

$\begin{array}{lllllllll}\text { Strongly Disagree } & 1 & 2 & 3 & 4 & 5 & 6 & 7 & \text { Strongly Agree }\end{array}$

\section{Control}

1. When navigating this website, I felt in control.

2. I felt that I had no control over my interaction with the Web.

3. This website allowed me to control the computer interaction.

\section{Attention focus}

1. When navigating this website, I thought about other things.

2. When navigating this website, I was aware of distractions.

3. When navigating this website, I was totally absorbed in what I was doing.

\section{Curiosity}

1. Navigating this website excited my curiosity.

2. Interacting with this website made me curious.

3. Navigating this website aroused my imagination. 
Intrinsic interest

1. Navigating this website bored me.

2. Navigating this website was intrinsically interesting.

3. This website was fun for me to use.

The Web performance measure

Please rate the performance of the website you most frequently visited in terms of your evaluation of the site or your feeling toward the site using the following terms.

The utilitarian performance

1. Ordered-chaotic

2. Wise-foolish

3. Reliable-unreliable

4. Effective-ineffective

5. Correct-wrong

The hedonic performance

1. Pleasant-unpleasant

2. Nice-awful

3. Entertaining-weary

4. Agreeable-disagreeable

5. Soothing-aggravating

\section{References}

Albers-Miller, N. D., \& Stafford, M. R. (1999). International services advertising: an examination of variation in appeal use for experiential and utilitarian services. Journal of Service Marketing, 13(4/5), 390-406.

Batra, R., \& Ahtola, O. T. (1990). Measuring the hedonic and utilitarian sources of consumer attitudes. Marketing Letters, 2(2), 159-170.

Bellman, S., Lohse, G. L., \& Johnson, E. J. (1999). Predictors of online buying behavior. Communications of the ACM, 42, 32-38.

Berlyne, D. E., Craw, M. A., \& Salapatek, P. H. (1963). Novelty, complexity, incongruity, extrinsic motivation, and the GSR. Journal of Experimental Psychology, 66, 560-567.

Bianchi, M. (1998). Consuming novelty: strategies for producing novelty in consumption. Journal of Medieval and Early Modern Studies, 28(1), 3-18.

Bryant, J., \& Love, C. (1996). Entertainment as the driver of new information technology. In R. R. Dholakia, N. Mundorf, \& N. Dholakia (Eds.), New infotainment technologies in the home: demand-side perspective (pp. 91-114). Mahwah, NJ: Lawrence Erlbaum Associates. 
Burgoon, J. K., Bonito, J. A., Bengtsson, B., \& Ramirez Jr., A. (1999/2000). Testing the interactivity model: communication processes, partner assessments, and the quality of collaborative work. Journal of Management Information Systems 16(3), 33-56.

Campbell, D. J. (1988). Task complexity: a review and analysis. Academy of Management Review, 13(1), 40-52.

Chen, H., Wigand, R. T., \& Nilan, M. S. (1999). Optimal experience of web activities. Computers in Human Behavior, 15(5), 585-608.

Crowley, A. E., Spangenberg, E. R., \& Hughes, K. R. (1992). Measuring the hedonic and utilitarian dimensions of attitudes toward product categories. Marketing Letters, 3(3), 239-249.

Csikszentmihalyi, M. (1975). Beyond boredom and anxiety. San Francisco: Jossey-Bass.

Davis, F. D., Bagozzi, R. P., \& Warshaw, P. R. (1992). Extrinsic and intrinsic motivation to use computers in the workplace. Journal of Applied Social Psychology, 22(14), 1111-1132.

Donovan, R. J., \& Rossiter, J. R. (1982). Store atmosphere: an experimental psychology approach. Journal of Retailing, 58, 34-57.

Eliashberg, J., \& Sawhney, M. S. (1994). Modeling goes to Hollywood: predicting individual differences in movie enjoyment. Management Science, 40(9), 1151-1173.

Ghani, J. A., \& Deshpande, S. P. (1994). Task characteristics and the experience of optimal flow in human-computer interaction. The Journal of Psychology, 128(4), 381-391.

Ha, L., \& James, E. L. (1998). Interactivity reexamined: a baseline analysis of early business web sites. Journal of Broadcasting \& Electronic Media, 42(4), 457-474.

Hammond, K., McWilliam, G., \& Diaz, A. N. (1998). Fun and work on the Web: differences in attitudes between novices and experienced users. Advances in Consumer Research, 25, 372-378.

Hoffman, D. L., \& Novak, T. P. (1996). Marketing in hypermedia computer-mediated environments: conceptual foundations. Journal of Marketing, 60, 50-68.

Hwang, M. I., \& Lin, J. W. (1999). Information dimension, information overload and decision quality. Journal of Information Science, 25(3), 213-219.

Igbaria, M., Schiffman, S. J., \& Wieckowski, T. J. (1994). The respective roles of perceived usefulness and perceived fun in the acceptance of microcomputer technology. Behavior \& Information Technology, 13(6), 349-361.

Iselin, E. R. (1988). The effects of information load and information diversity on decision quality in a structured decision task. Accounting, Organizations and Society, 13(2), 147-165.

Kahneman, D. (1973). Attention and effort. Englewood Cliffs, NJ: Prentice-Hall.

Loewenstein, G. (1994). The psychology of curiosity: a review and reinterpretation. Psychological Bulletin, 116(1), 75-98.

Mehrabian, A., \& Russell, J. A. (1974). An approach to environmental psychology. Cambridge, MA: MIT Press.

Novak, T. P., Hoffman, D. L., \& Yung, Y. F. (2000). Measuring the customer experience in online environments: a structural modeling approach. Marketing Science, 19, 22-42.

Rogers, E. M. (1995). Diffusion of innovations. New York: Free Press.

Posnock, R. (1991). The trail of curiosity: Henry James, William James, and the challenge of modernity. New York: Oxford University Press.

Privette, G. (1983). Peak experience, peak performance, and flow: a comparative analysis of positive human experiences. Journal of Personality and Social Psychology, 45(6), 1361-1368.

Singh, S. N., \& Dalal, N. P. (1999). Web home pages as advertisements. Communications of the ACM, 42(8), 91-98.

Srinivasan, T. C. (1987). An integrative approach to consumer choice. Advances in Consumer Research, 14, 96-100.

Steenkamp, J. E. M., \& Baumgartner, H. (1992). The role of optimum stimulation level in exploratory consumer behavior. Journal of Consumer Research, 19(3), 434-448.

Stell, R., \& Paden, N. (1999). Vicarious exploration and catalog shopping: a preliminary investigation. The Journal of Consumer Marketing, 16(4), 332-346.

Trevino, L. K., \& Webster, J. (1992). Flow in computer-mediated communication. Communication Research, 19(5), 539-573.

Venkatesh, V. (2000). Determinants of perceived ease of use: integrating control, intrinsic motiva- 
tion, and emotion into the technology acceptance model. Information Systems Research, 11(4), $342-365$.

Webster, J., Trevino, L. K., \& Ryan, L. (1993). The dimensionality and correlates of flow in humancomputer interactions. Computers in Human Behavior, 9, 411-426.

Willcocks, L. P., \& Plant, R. (2001). Pathways to e-business leadership: getting from bricks and clicks. MIT Sloan Management Review, 42, 50-59.

Williams, R. L., \& Cothrel, J. (2000). Four smart ways to run online communities. MIT Sloan Management Review, 4, 81-91.

Wolfinbarger, M., \& Gilly, M. C. (2001). Shopping online for freedom, control, and fun. California Management Review, 43, 34-55. 